\title{
Preliminary evaluation of circulating microRNAs as potential biomarkers in paracoccidioidomycosis
}

\author{
JUNYA DE LACORTE SINGULANI $^{1}$, JULHIANY DE FÁTIMA DA SILVA ${ }^{1}$, \\ FERNANDA PATRICIA GULLO ${ }^{1}$, MARINA CÉLIA COSTA ${ }^{2}$, ANA MARISA FUSCO-ALMEIDA ${ }^{1}$, \\ FRANCISCO JAVIER ENGUITA $^{2}$ and MARIA JOSÉ SOARES MENDES-GIANNINI ${ }^{1}$ \\ ${ }^{1}$ Department of Clinical Analysis, School of Pharmaceutical Sciences, São Paulo State University (UNESP), \\ Araraquara, São Paulo 14800-901, Brazil; ${ }^{2}$ Faculty of Medicine, Institute of Molecular Medicine, \\ University of Lisbon, 1649-004 Lisbon, Portugal
}

Received September 26, 2016; Accepted January 9, 2017

DOI: $10.3892 / b r .2017 .849$

\begin{abstract}
MicroRnAs (miRNAs) are small RNAs (length, 19-24 nucleotides) that regulate gene expression by either mRNA degradation or translational inhibition of proteins. Circulating miRNAs, which are extremely stable and protected from RNAse-mediated degradation in body fluids, have appeared as candidate biomarkers for numerous diseases. However, little is known about circulating miRNAs in fungal infections. Paracoccidioidomycosis (PCM) is caused by the Paracoccidioides species, and is endemic in Central and South America, with predominance in adult male workers from rural areas. The current study aimed to identify a serum miRNA expression profile that could serve as a novel diagnostic biomarker for PCM. Total RNA was isolated and the levels of circulating miRNAs were compared between patients with PCM and healthy control subjects using reverse transcription-quantitative polymerase chain reaction. Bioinformatic analysis was used to evaluate the potential roles of these miRNAs in PCM. Eight miRNAs were differentially expressed in serum samples from patients with PCM. These miRNAs are associated with apoptosis and immune response. The identified miRNAs facilitate with understanding the regulatory mechanisms involved in the host-parasite interaction of PCM. Furthermore, considering that the diagnosis of PCM presents difficulties, these miRNAs may serve as novel biomarkers for this disease.
\end{abstract}

Correspondence to: Dr Maria José Soares Mendes-Giannini, Department of Clinical Analysis, School of Pharmaceutical Sciences, São Paulo State University (UNESP), Rodovia Araraquara Jaú Km 1, Araraquara, São Paulo 14800-901, Brazil

E-mail: gianninimj@gmail.com

Key words: microRNA, serum, Paracoccidioides spp., biological function, biomarker

\section{Introduction}

MicroRNAs (miRNAs) are an abundant class of short regulatory non-coding RNAs (ncRNAs; length, 20-24 nucleotides) that act as post-transcriptional repressors by binding the 3'-untraslated regions (UTRs) of target messenger RNAs (mRNAs). The regulatory activity of miRNAs is exerted at the post-transcriptional level and it is estimated that they act on up to one-third of the protein coding genes. miRNAs have been implicated in various biological and pathological processes, and have emerged as important contributors to cell homeostasis (1).

Cells actively secrete miRNAs, and it is possible to detect those small ncRNAs in all biological fluids. As a consequence, circulating miRNAs have great potential as biomarkers, which has already been reported for various types of human disease, such as cancer (2), metabolic disorders (3) and cardiac conditions (4). In systemic infections, the presence of a pathogenic agent often induces a significant change in the profile of circulating miRNAs that facilitates their use as biomarkers of disease establishment and progression. In certain cases, such as viral infections, these changes in circulating miRNAs are associated with the targeted cell, as demonstrated in the acute and chronic hepatitis $C$ virus infection $(5,6)$. Parasitic flatworms, such as Schistosoma japonicum induce a differential expression of circulating miRNAs (7). Furthermore, previous studies have suggested the involvement of circulating miRNAs in response to bacterial infections, including active pulmonary tuberculosis (8). However, the potential use of circulating miRNAs as biomarkers in fungal infections has been investigated comparatively less. In this context, in response to infection with Candida albicans, miR-455, miR-125a, miR-146 and miR-155 were upregulated in mouse macrophages and miR-204 and miR-211 were downregulated in renal mice tissues $(9,10)$. In monocytes and dendritic cells infected with Aspergillus fumigatus, miR-132 and miR-155 were identified to be differentially expressed (11).

The incidence of fungal infections has increased in recent years and is associated with significant morbidity and mortality (12). Paracoccidioidomycosis (PCM) is a human systemic mycosis that is considered to be clinically important, due to the increase of frequency, the severity of their clinical 
manifestations and the associated mortality rates. PCM is caused by dimorphic fungus of the Paracoccidioides species (Paracoccidioides brasiliensis and Paracoccidioides lutzii) and is endemic in Central and South America, with a predominance of clinical disease in adult men and labourers from rural areas (13). In patients with PCM, the fungus is initially observed in the lungs; however, the yeast spreads to other organs (14).

Considering the central role of miRNAs in infectious diseases and that little is known about the molecular mechanisms underlying PCM, the aim of the present study was to verify the presence of differentially expressed circulating miRNAs in patients with PCM, which could serve as novel biomarkers in the diagnosis of this fungal disease.

\section{Materials and methods}

Study subjects. Four male patients diagnosed with chronic PCM, and four male healthy control subjects free of the PCM infection and free of any clinical symptoms of any infectious disease were recruited from the School of Pharmaceutical Sciences, São Paulo State University (UNESP; Araraquara, Brazil) and included in the current study. Venous blood $(1 \mathrm{ml})$ was collected from each participant and samples were centrifuged at $1,800 \mathrm{x}$ g for $10 \mathrm{~min}$ at $4^{\circ} \mathrm{C}$. The supernatant serum was quickly removed, aliquoted, and frozen at $-80^{\circ} \mathrm{C}$ until the experiments were performed. The present study was approved by the local ethics committee of the School of Pharmaceutical Sciences, São Paulo State University (UNESP) (approval no. 33397114.5.0000.5426) and was performed in accordance with the Declaration of Helsinki. Written informed consent was obtained prior to blood sample collection.

Extraction of RNA. The extraction of RNA, including miRNAs, from $200 \mu \mathrm{l}$ serum was performed using miRCURY'T RNA Isolation kit - Biofluids (Exiqon A/S, Vedbaek, Denmark), according to the manufacturer's instructions.

Synthesis of cDNA and analysis by reverse transcription-quantitative polymerase chain reaction (RT-qPCR). cDNA was synthesized from RNA using a Universal cDNA synthesis kit (Exiqon A/S) according to the manufacturer's instructions. For miRNA screening, a global RT-qPCR low-density panel (microRNA Ready-to-Use PCR, Human panel I+II, V.2 M/R; Exiqon A/S) was used, which contains locked nucleic acid specific primers for 752 unique human miRNAs. The reaction mixture included $40 \mu 1 \mathrm{cDNA}, 40$ $\mathrm{ml} \mathrm{SYBR-Green} \mathrm{PCR} \mathrm{Master} \mathrm{Mix} \mathrm{(Exiqon} \mathrm{A/S)} \mathrm{and} 40 \mathrm{ml}$ RNase-free water. Then, $10 \mu \mathrm{l}$ of the reaction mixture was added to each well of the panel. Amplification was performed by RT-qPCR (ViiA ${ }^{\mathrm{TM}} 7$ Real-Time PCR System; Thermo Fisher Scientific, Inc., Waltham, MA, USA) followed by the determination of the melting curve according the following conditions: Denaturation at $95^{\circ} \mathrm{C}$ for $10 \mathrm{~min}$, followed by 45 cycles of $95^{\circ} \mathrm{C}$ for $10 \mathrm{sec}$ and $60^{\circ} \mathrm{C}$ for $60 \mathrm{sec}$. The experiment was performed in triplicate. The raw $\mathrm{Cq}$ values were normalized using the global normalization method in each sample, and the expression levels of all miRNAs were calculated according to the $\Delta \Delta \mathrm{Cq}$ method (15), as implemented in

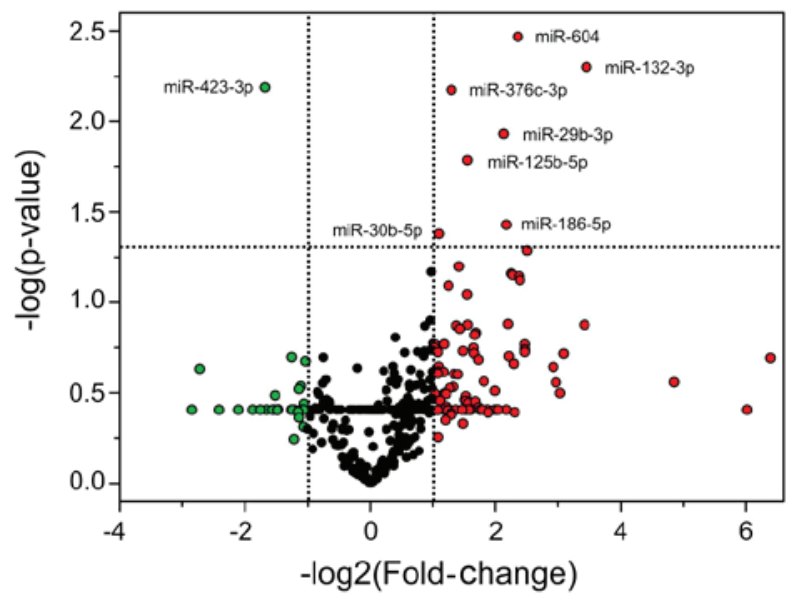

Figure 1. Volcano plot of the detected circulating miRNAs in patients with paracoccidioidomycosis compared with healthy controls. Upregulated and downregulated miRNAs are presented in red and green, respectively. Statistically significant miRNAs are depicted with their miRBase names. miRNA, microRNA.

DataAssist v2 software (Applied Biosystems; Thermo Fisher Scientific, Inc.).

Statistical analysis. The statistics toolbox of the DataAssist v2 software (Applied Biosystems; Thermo Fisher Scientific, Inc.) was used and $\mathrm{P}<0.05$ was considered to indicate a statistically significant difference. A volcano plot was constructed for the analyzed miRNAs and miRBase was used to identify them (16). For bioinformatics analysis, differentially expressed miRNAs were selected and submitted to in silico pathways analysis for Gene Ontology (GO) annotation and Kyoto Encyclopedia of Genes and Genomes (KEGG), using the bioinformatics tool, Database for Annotation, Visualization and Integrated Discovery (DAVID).

\section{Results}

Circulating miRNA levels in the PCM. The differentially expressed miRNAs in PCM patients are demonstrated by a non-symmetrical distribution in a volcano plot (Fig. 1). The $\mathrm{x}$-axis represents the $\log _{2}$ ratio of the expression difference of miRNA between the groups and the y-axis demonstrates the $\log$ of the P-values. From the 752 miRNAs analyzed, seven were overexpressed (miR-132-3p, miR-604, miR-186-5p, miR-29b-3p, miR-125b-5p, miR-376c-3p and miR-30b-5p) and one was underexpressed (miR-423-3p) in the serum of patients with PCM when compared with the healthy control subjects (Table I). The data were regarded as significantly different at $\mathrm{P}<0.05$ and with a fold-change $\geq 2$. Furthermore, the expression profile of the investigated microRNAs was similar among the subjects of each group.

Pathway analysis of miRNA-controlled pathways. Based on the prediction of pathways, using the gene annotation tool DAVID (Table II), the differentially expressed miRNAs in PCM participate in the processes of apoptosis, immune response, adhesion and the infection processes of fungus to host cells. The biological processes identified may regulate genes that are included in the mitogen-activated protein kinases 
Table I. Differentially expressed miRNAs in patients with paracoccidioidomycosis relative to healthy control subjects.

\begin{tabular}{lcc}
\hline miRNA & Fold-change & P-value \\
\hline Upregulated & & \\
hsa-miR-132-3p & 10.97 & 0.0050 \\
hsa-miR-604 & 5.13 & 0.0034 \\
hsa-miR-186-5p & 4.51 & 0.0372 \\
hsa-miR-29b-3p & 4.38 & 0.0117 \\
hsa-miR-125b-5p & 2.93 & 0.0164 \\
hsa-miR-376c-3p & 2.45 & 0.0067 \\
hsa-miR-30b-5p & 2.14 & 0.0418 \\
Downregulated & & \\
hsa-miR-423-3p & 0.31 & 0.0065 \\
\hline
\end{tabular}

miRNA, microRNA.

Table II. Signaling pathways regulated by eight microRNAs identified in patients with paracoccidioidomycosis.

\begin{tabular}{ll}
\hline Signaling pathway & P-value \\
\hline Regulation of apoptosis & $1.8 \mathrm{E}-28$ \\
Negative regulation of transcription & $3.2 \mathrm{E}-16$ \\
Metabolism regulation of cellular proteins & $2.3 \mathrm{E}-18$ \\
Regulation of DNA binding & $1.7 \mathrm{E}-18$ \\
Regulation of the cascade of protein kinases & $3.8 \mathrm{E}-12$ \\
Regulation of cell migration & $8.9 \mathrm{E}-15$ \\
Inflammatory response & $2.3 \mathrm{E}-8$ \\
Mitogen activated protein kinases cascade & $2.4 \mathrm{E}-5$ \\
Activation of leukocytes & $1.1 \mathrm{E}-9$ \\
Activation of T cells & $4.5 \mathrm{E}-6$ \\
Activation of B cells & $2.7 \mathrm{E}-3$ \\
Regulation of cytokine production & $8.5 \mathrm{E}-10$ \\
Regulation of adhesion to the extracellular matrix & $8.2 \mathrm{E}-7$ \\
Endocytosis & $2.7 \mathrm{E}-5$ \\
Binding to the tumor necrosis factor receptor & $3.6 \mathrm{E}-6$ \\
superfamily &
\end{tabular}

cascade, inflammatory response, activation of leukocytes, $\mathrm{T}$ and $\mathrm{B}$ cells, regulation of cytokine production, regulation of adhesion to the extracellular matrix (ECM) and endocytosis.

\section{Discussion}

Previous studies have shown the involvement of circulating miRNAs in certain infectious and non-infectious diseases $(2,8,17,18)$. miRNAs may be used for diagnosis, as well as for monitoring disease progression and treatment efficacy (19). This is a preliminary study that compared the miRNA profile in the serum of patients diagnosed with chronic PCM with healthy control subjects. Eight miRNAs (miR-132-3p, miR-604, miR-186-5p, miR-29b-3p, miR-125b-5p, miR-376c-3p, miR-30b-5p and miR-423-3p) were identified to be differentially expressed in serum samples obtained from patients with PCM.

The identification of miRNAs differentially expressed in PCM in the present study is considered to be important. It facilitates the understanding of the regulatory mechanisms involved in host-parasite interactions and the infectious process. The in silico analysis demonstrated that apoptosis, immune response and adhesion of fungus to host cells are important signaling pathways that are regulated by eight differentially expressed miRNAs in PCM. Following inhalation by the host, Paracoccidioides spp. adhere to ECM components, such as collagen, fibronectin and laminin. This process avoids elimination of the fungi by lung ciliary cells and contributes to establishment of the infection (20). Apoptosis is an important mechanism, as described in certain infections, including PCM, and has been implicated in immunosuppressive events, which works in favor of the parasite to limit the exaggerated inflammatory response. The process of apoptosis has been described in PCM patients and in a PCM murine model $(21,22)$. P. brasiliensis induces apoptosis in macrophages via caspases $(23,24)$. In addition, the participation of macrophages, cytokines, interleukins (ILs) and tumor necrosis factor (TNF) is observed in PCM $(20,25)$.

In addition, the majority of miRNAs identified in the present study have been previously described in the processes of apoptosis and immune response to other infections. miR-132 and miR-186, for example, have been described to be associated with the promotion of apoptosis, which activates the signaling pathway via caspases (26). Furthermore, the expression of miR-132 was induced in cells of the immune system by the A.fumigatus and Mycobacterium tuberculosis infection $(11,27)$. Members of the miR-29 family may act in the Wnt signaling pathway, which regulates the activation of macrophages in the inflammatory process $(28,29)$. Furthermore, miR-29 was identified to be overexpressed in the serum and sputum of patients with active pulmonary tuberculosis (8). Comparable with miR-29, miR-376c was recently reported to be differentially expressed in the serum of patients with active tuberculosis (30). In addition, miR-125 may be involved in the immune response to infections. It has been shown that $M$. tuberculosis induces the expression of miR-125b, which binds to the 3'-UTR of the mRNA of TNF in human macrophages and destabilizes the transcript. Blocking biosynthesis of the TNF is an important process in response to bacterial infection (31). miR-30 may also be associated with the immune response process, as it suppresses the polypeptide $N$-acetylgalactosaminyltransferase 7 gene, which leads to an increase of IL-10 (32). By contrast, two miRNAs (has-miR-604 and $\mathrm{miR}-423-3 \mathrm{p}$ ) were described, but were not associated with infectious disease $(33,34)$.

miRNAs detected in the serum of patients with PCM may serve as biomarkers. The diagnosis of PCM is based on clinical and laboratory findings. Typically, the direct microscopic examination of lesions or tissue samples that are collected is made with $10 \%$ potassium hydroxide, $4 \%$ sodium hydroxide or calcofluor white to observe the fungi. The culture to observe the thermal dimorphism of Paracoccidioides spp. is necessary for a definitive diagnosis; however, the fungus grows slowly. Serologic tests facilitate with diagnosis, however, certain tests for PCM are not well standardized, which may 
result in cross-reactions with other infections (35). Thus, the production of antigenic preparations from whole yeast cells and culture filtrate is important to ensure the sensitivity and specificity of the tests. Gp43 has served as the main antigen in the diagnosis of PCM; however, it was demonstrated that high percentages of false negative results for PCM were caused by $P$. lutzii (36). Thus, novel biomarkers along with existing techniques may contribute to improved diagnosis of PCM. In this context, miRNAs have been presented as 'fingerprints' of various pathological conditions (37). These molecules are advantageous due to their stability in body fluids, as they are normally packaged into exosome-like microparticles that protect them against endogenous RNase activity. Circulating miRNAs have been shown to be stable during repeated freezing and thawing, as well as in acidic and alkaline environments (38). Furthermore, detection techniques, including PCR and microarray, are sensitive and rapid $(1,39)$.

In conclusion, eight differentially expressed miRNAs were identified in serum samples from patients with PCM. These miRNAs are associated with apoptosis, immune responses and adhesion processes. Although the small sample size is a limitation, the present study provided, to the best of our knowledge, the first description of circulating miRNAs as potential biomarkers for the diagnosis of PCM. However, further studies are required to extend the identification of miRNAs for a larger number of patients with PCM and to clarify the role of theses miRNAs in this mycosis.

\section{Acknowledgements}

The present study was supported by grants from the Coordenação de Aperfeiçoamento de Pessoal de Nível Superior/Fundação para a Ciência e a Tecnologia (grant no. 345/13), the Conselho Nacional de Pesquisa e Desenvolvimento the Fundação de Amparo à Pesquisa do Estado de São Paulo (grant no. 2014/10446-9) and Programa de Apoio ao Desenvolvimento Científico da Faculdade de Ciências Farmacêuticas da UNESP.

\section{References}

1. Hu G, Drescher KM and Chen XM: Exosomal miRNAs: Biological properties and therapeutic potential. Front Genet 3: $56,2012$.

2. Liu X, Liu L, Xu Q, Wu P, Zuo X and Ji A: MicroRNA as a novel drug target for cancer therapy. Expert Opin Biol Ther 12: 573-580, 2012 .

3. Peng Y, Yu S, Li H, Xiang H, Peng J and Jiang S: MicroRNAs: Emerging roles in adipogenesis and obesity. Cell Signal 26: 1888-1896, 2014.

4. Goretti E, Zangrando J, Wagner DR and Devaux Y: Unity is strength - a panel of 4 microRNAs decreases cardiomyocyte hypertrophy. Int J Cardiol 182: 62-64, 2015.

5. El-Diwany R, Wasilewski LN, Witwer KW, Bailey JR, Page K, Ray SC, Cox AL, Thomas DL and Balagopal A: Acute Hepatitis $\mathrm{C}$ virus infection induces consistent changes in circulating microRNAs that are associated with nonlytic hepatocyte release. J Virol 89: 9454-9464, 2015.

6. Shrivastava S, Petrone J, Steele R, Lauer GM, Di Bisceglie AM and Ray RB: Up-regulation of circulating miR-20a is correlated with hepatitis $\mathrm{C}$ virus-mediated liver disease progression. Hepatology 58: 863-871, 2013.

7. Cai P, Gobert GN, You H, Duke M and McManus DP: Circulating miRNAs: Potential novel biomarkers for hepatopathology progression and diagnosis of schistosomiasis japonica in two murine models. PLoS Negl Trop Dis 9: e0003965, 2015.
8. Fu Y, Yi Z, Wu X, Li J and Xu F: Circulating microRNAs in patients with active pulmonary tuberculosis. J Clin Microbiol 49: 4246-4251, 2011.

9. Monk CE, Hutvagner G and Arthur JS: Regulation of miRNA transcription in macrophages in response to Candida albicans. PLoS One 5: e13669, 2010.

10. Li XY, Zhang K, Jiang ZY and Cai LH: miR-204/miR-211 downregulation contributes to candidemia-induced kidney injuries via derepression of Hmx1 expression. Life Sci 102: 139-144, 2014.

11. Das Gupta M, Fliesser M, Springer J, Breitschopf T, Schlossnagel H, Schmitt AL, Kurzai O, Hünniger K, Einsele H and Löffler J: Aspergillus fumigatus induces microRNA-132 in human monocytes and dendritic cells. Int J Med Microbiol 304: 592-596, 2014.

12. Pfaller MA and Diekema DJ: Epidemiology of invasive mycoses in North America. Crit Rev Microbiol 36: 1-53, 2010.

13. Shikanai-Yasuda MA, Telles Filho FeQ, Mendes RP, Colombo AL and Moretti ML: Guidelines in paracoccidioidomycosis. Rev Soc Bras Med Trop 39: 297-310, 2006 (In Portuguese).

14. Marques SA: Paracoccidioidomycosis. Clin Dermatol 30: 610-615, 2012.

15. Livak KJ and Schmittgen TD: Analysis of relative gene expression data using real-time quantitative PCR and the 2(-Delta Delta C(T)) method. Methods 25: 402-408, 2001.

16. Griffiths-Jones S, Grocock RJ, van Dongen S, Bateman A and Enright AJ: miRBase: microRNA sequences, targets and gene nomenclature. Nucleic Acids Res 34: D140-D144, 2006.

17. Reid G, Kirschner MB and van Zandwijk N: Circulating microRNAs: Association with disease and potential use as biomarkers. Crit Rev Oncol Hematol 80: 193-208, 2011.

18. Chen Y, Li L, Zhou Z, Wang N, Zhang CY and Zen K: A pilot study of serum microRNA signatures as a novel biomarker for occult hepatitis B virus infection. Med Microbiol Immunol (Berl) 201: 389-395, 2012.

19. Weiland M, Gao XH, Zhou L and Mi QS: Small RNAs have a large impact: Circulating microRNAs as biomarkers for human diseases. RNA Biol 9: 850-859, 2012.

20. de Oliveira HC, Assato PA, Marcos CM, Scorzoni L, de Paula E Silva AC, Da Silva JF, Singulani JL, Alarcon KM, Fusco-Almeida AM and Mendes-Giannini MJ: Paracoccidioides-host Interaction: An overview on recent advances in the Paracoccidioidomycosis. Front Microbiol 6: 1319, 2015.

21. Cacere CR, Romano CC, Mendes Giannini MJ, Duarte AJ and Benard G: The role of apoptosis in the antigen-specific $\mathrm{T}$ cell hyporesponsiveness of paracoccidioidomycosis patients. Clin Immunol 105: 215-222, 2002

22. Verícimo MA, França KM, Arnholdt AC and Kipnis TL: Increased apoptosis during the early phase of experimental paracoccidioidomycosis as a phenotypic marker of resistance. Microbes Infect 8: 2811-2820, 2006.

23. Silva SS, Tavares AH, Passos-Silva DG, Fachin AL, Teixeira SM, Soares CM, Carvalho MJ, Bocca AL, Silva-Pereira I, Passos GA and Felipe MS: Transcriptional response of murine macrophages upon infection with opsonized Paracoccidioides brasiliensis yeast cells. Microbes Infect 10: 12-20, 2008.

24. Del Vecchio A, Silva JF, Silva JL, Andreotti PF, Soares CP, Benard G and Giannini MJ: Induction of apoptosis in A549 pulmonary cells by two Paracoccidioides brasiliensis samples. Mem Inst Oswaldo Cruz 104: 749-754, 2009.

25. Fortes MR, Miot HA, Kurokawa CS, Marques ME and Marques SA: Immunology of paracoccidioidomycosis. An Bras Dermatol 86: 516-524, 2011.

26. Zhang B, Lu L, Zhang X, Ye W, Wu J, Xi Q and Zhang X: hsa-miR-132 regulates apoptosis in non-small cell lung cancer independent of acetylcholinesterase. J Mol Neurosci 53: 335-344, 2014.

27. Ni B, Rajaram MV, Lafuse WP, Landes MB and Schlesinger LS: Mycobacterium tuberculosis decreases human macrophage IFN- $\gamma$ responsiveness through miR-132 and miR-26a. J Immunol 193: 4537-4547, 2014

28. Kapinas K, Kessler C, Ricks T, Gronowicz G and Delany AM: miR-29 modulates Wnt signaling in human osteoblasts through a positive feedback loop. J Biol Chem 285: 25221-25231, 2010.

29. Pereira CP, Bachli EB and Schoedon G: The wnt pathway: A macrophage effector molecule that triggers inflammation. Curr Atheroscler Rep 11: 236-242, 2009.

30. Zhang H, Sun Z, Wei W, Liu Z, Fleming J, Zhang S, Lin N, Wang M, Chen M, Xu Y, et al: Identification of serum microRNA biomarkers for tuberculosis using RNA-seq. PLoS One 9: e88909, 2014. 
31. Rajaram MV, Ni B, Morris JD, Brooks MN, Carlson TK, Bakthavachalu B, Schoenberg DR, Torrelles JB and Schlesinger LS: Mycobacterium tuberculosis lipomannan blocks TNF biosynthesis by regulating macrophage MAPK-activated protein kinase 2 (MK2) and microRNA miR-125b. Proc Natl Acad Sci USA 108: 17408-17413, 2011.

32. Gaziel-Sovran A, Segura MF, Di Micco R, Collins MK, Hanniford D, Vega-Saenz de Miera E, Rakus JF, Dankert JF, Shang S, Kerbel RS, et al: miR-30b/30d regulation of GalNAc transferases enhances invasion and immunosuppression during metastasis. Cancer Cell 20: 104-118, 2011.

33. Sabina S, Pulignani S, Rizzo M, Cresci M, Vecoli C, Foffa I, Ait-Ali L, Pitto L and Andreassi MG: Germline hereditary, somatic mutations and microRNAs targeting-SNPs in congenital heart defects. J Mol Cell Cardiol 60: 84-89, 2013.

34. Guan G, Zhang D, Zheng Y, Wen L, Yu D, Lu Y and Zhao Y: microRNA-423-3p promotes tumor progression via modulation of AdipoR2 in laryngeal carcinoma. Int J Clin Exp Pathol 7: 5683-5691, 2014.
35. Caldini CP, Xander P, Kioshima ÉS, Bachi AL, de Camargo ZP, Mariano M and Lopes JD: Synthetic peptides mimic gp75 from Paracoccidioides brasiliensis in the diagnosis of paracoccidioidomycosis. Mycopathologia 174: 1-10, 2012.

36. Batista J Jr, de Camargo ZP, Fernandes GF, Vicentini AP, Fontes CJ and Hahn RC: Is the geographical origin of a Paracoccidioides brasiliensis isolate important for antigen production for regional diagnosis of paracoccidioidomycosis? Mycoses 53: 176-180, 2010.

37. Polakovičová M, Musil P, Laczo E, Hamar D and Kyselovič J: Circulating microRNAs as potential biomarkers of exercise response. Int J Mol Sci 17: E1553, 2016.

38. Kim T and Reitmair A: Non-Coding RNAs: Functional aspects and diagnostic utility in oncology. Int J Mol Sci 14: 4934-4968, 2013.

39. Madhavan D, Cuk K, Burwinkel B and Yang R: Cancer diagnosis and prognosis decoded by blood-based circulating microRNA signatures. Front Genet 4: 116, 2013. 